\title{
Perfect Orientation Ordered in-situ One-Dimensional- Self-Assembly of Mn-doped PbSe Nanocrystals
}

Weigang Lu ${ }^{1}$, Puxian Gao ${ }^{2}$, Wen Bin Jian ${ }^{3}$, Zhong Lin Wang ${ }^{2}$ and Jiye Fang ${ }^{\text {* }}$

${ }^{1}$ Department of Chemistry and Advanced Materials Research Institute,

University of New Orleans, New Orleans, LA 70148, USA

${ }^{2}$ School of Materials Science and Engineering, Georgia Institute of Technology,

Atlanta, GA 30332-0245, USA

${ }^{3}$ Department of Physics, National Chung Hsing University, 250 Kuo Kwang Road, Taichung 40254, Taiwan, ROC

\section{Supplementary Information}



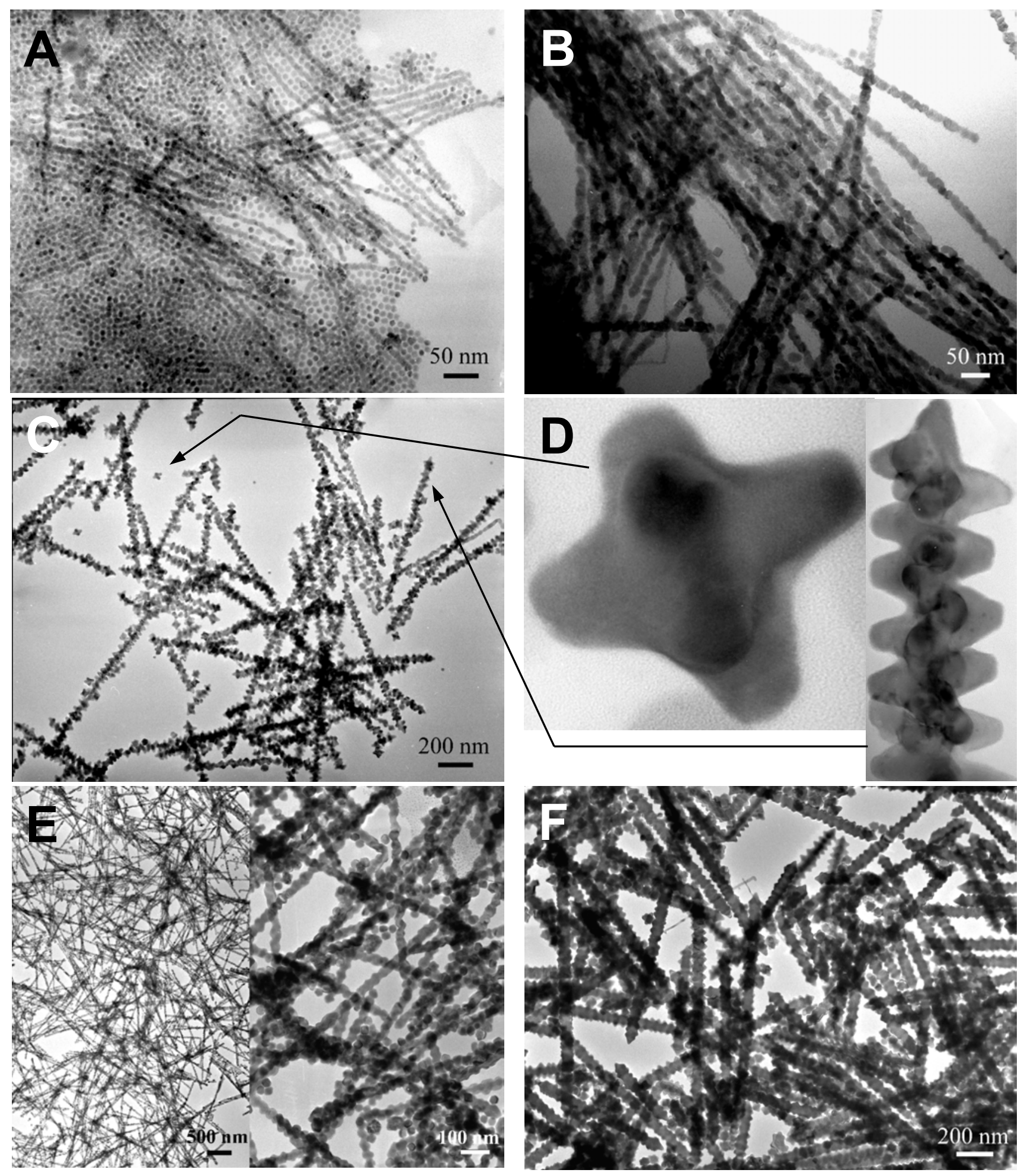

Figure S1. Transmission electron micrographs of $\mathrm{Pb}_{0.996} \mathrm{Mn}_{0.004} \mathrm{Se}$ corrugated nanoarrays self-assembled under various conditions. A, $180{ }^{\circ} \mathrm{C}$ for $5 \mathrm{~min}$; $\mathrm{B}, 200{ }^{\circ} \mathrm{C}$ for $5 \mathrm{~min}$; $\mathrm{C}$ and $\mathrm{D}$, $200{ }^{\circ} \mathrm{C}$ for $20 \mathrm{~min}$; E, $250{ }^{\circ} \mathrm{C}$ for $5 \mathrm{~min}$; F, $250{ }^{\circ} \mathrm{C}$ for $20 \mathrm{~min}$. Images were taken using a JEOL 2010 transmission electron microscope. 
Figure $\mathrm{S} 1$ illustrates the TEM images of $1 \mathrm{D} \mathrm{Pb}_{0.996} \mathrm{Mn}_{0.004} \mathrm{Se}(\mathrm{PbMnSe})$ corrugated nanoarrays assembled for $5 \mathrm{~min}$ and for $20 \mathrm{~min}$ at various temperatures. This observation reveals that the trace of $\mathrm{PbMnSe}$ corrugated nanoarrays is detectable when the sample is selfassembled at $180{ }^{\circ} \mathrm{C}$ for $5 \mathrm{~min}$ although some individual $\mathrm{NCs}$ are still considered as a dominant phase (Figure S1-A). The average diameter of these corrugated nanoarrays is less than $10 \mathrm{~nm}$. It has also been observed that, at growth temperature of $180{ }^{\circ} \mathrm{C}$, most portions of those assembled NCs are crystal orientation ordered and all the NCs obtained including those of individual ones are highly crystalline (not shown). When the in-situ self-assembly temperature is raised to $200{ }^{\circ} \mathrm{C}, 1 \mathrm{D}$ corrugated nanoarrays dominates over the products (Figures S1-B and S1-C) although a few of free single crystal elements can still be detected (Figure S1-D). Furthermore, an increase of evolution time from $5 \mathrm{~min}$ to $20 \mathrm{~min}$ results in assembly shape changes from single rows of stacked NCs enclosed by corrugated surfaces (diameter: $\sim 15 \mathrm{~nm}$ ) to multi-lines of stacked octahedra along the central axis with zigzag surface features, but no increase in length. In the case of the multi-line nanoarrays, the transverse diameter of assembled corrugated nanoarrays (i.e. the diagonal of the octahedron which is perpendicular to the stacking axis) is estimated as $\sim 30 \mathrm{~nm}$. This observation is in agreement with our previous report, ${ }^{1}$ i.e. a polyhedral surface is normally associated with crystals smaller than 10-20 nm which possibly results in a higher surface energy. The evolution of geometrical shapes for cubooctahedral NCs is depending on the ratio, $\mathrm{R}$, of the growth rate along the $<100>$ to that of the $<111>$ directions. By increasing the assembly time (or temperature), the area ratio of $\{111\}$ to $\{100\}$ is increased, resulting in an evolution of crystal shape from a triangle-based pyramid to a tetrahedron or octahedron. When $\mathrm{R}$ reaches to 1.73 , octahedral NCs as the building elements of the corrugated nanoarrays are perfectly formed. A free single octahedral NC demonstrated in Figure S1-D is an evidence to strongly support this suggestion. As showed in Figure S2, well-developed PbMnSe corrugated nanoarrays can be retrieved when the assembly temperature is further increased to $250{ }^{\circ} \mathrm{C}$, which is close to the b.p. of phenyl ether. These SEM images reveal that the longer the selfassembly time elapses, the thicker the arrays grow. The diameter of these corrugated nanoarrays assembled at $250{ }^{\circ} \mathrm{C}$ is estimated from $\sim 30 \mathrm{~nm}$ to $\sim 80 \mathrm{~nm}$ depending on the particular assembly time and respective growth modes. One of the typical XRD patterns recorded from these $\mathrm{PbMnSe}$ corrugated nanoarrays is also presented in Figure S2-F, indicating that it matches the bulk rock-salt structure of $\mathrm{PbSe}$ well. 

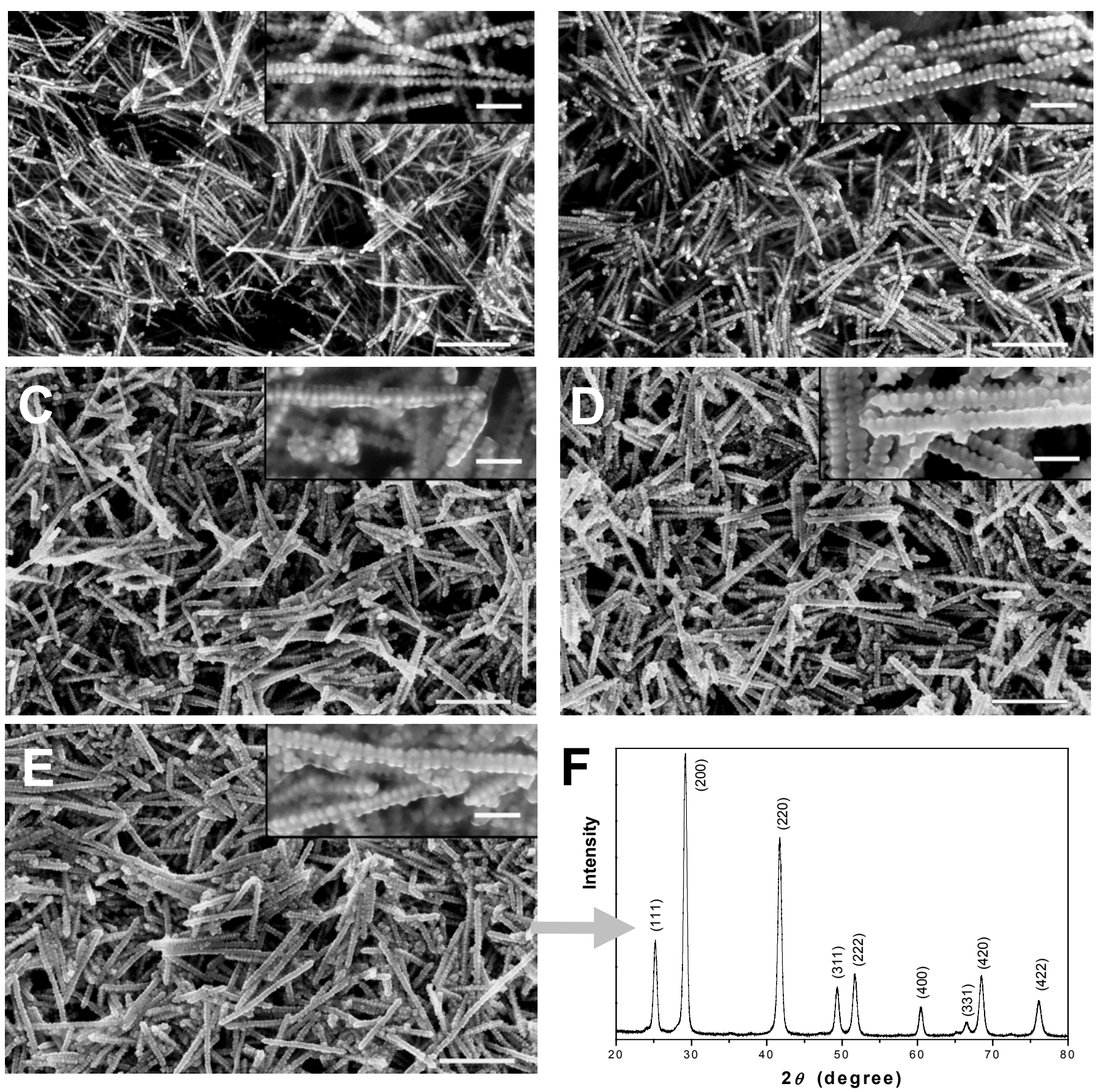

Figure S2. Scanning electron micrographs of $\mathrm{Pb}_{0.996} \mathrm{Mn}_{0.004}$ Se corrugated nanoarrays self-assembled at $250{ }^{\circ} \mathrm{C}$ for (A) $5 \mathrm{~min}$; (B) $10 \mathrm{~min}$; (C) $15 \mathrm{~min}$; (D) $20 \mathrm{~min}$ and (E) $25 \mathrm{~min}$; bar at bottom, $1 \mu \mathrm{m}$; bar in inset, $200 \mathrm{~nm}$. Pattern (F) is a powder x-ray diffraction trace of the $\mathrm{Pb}_{0.996} \mathrm{Mn}_{0.004} \mathrm{Se}$ nanoarrays in-situ self-assembled at $250{ }^{\circ} \mathrm{C}$ for $25 \mathrm{~min}$. 


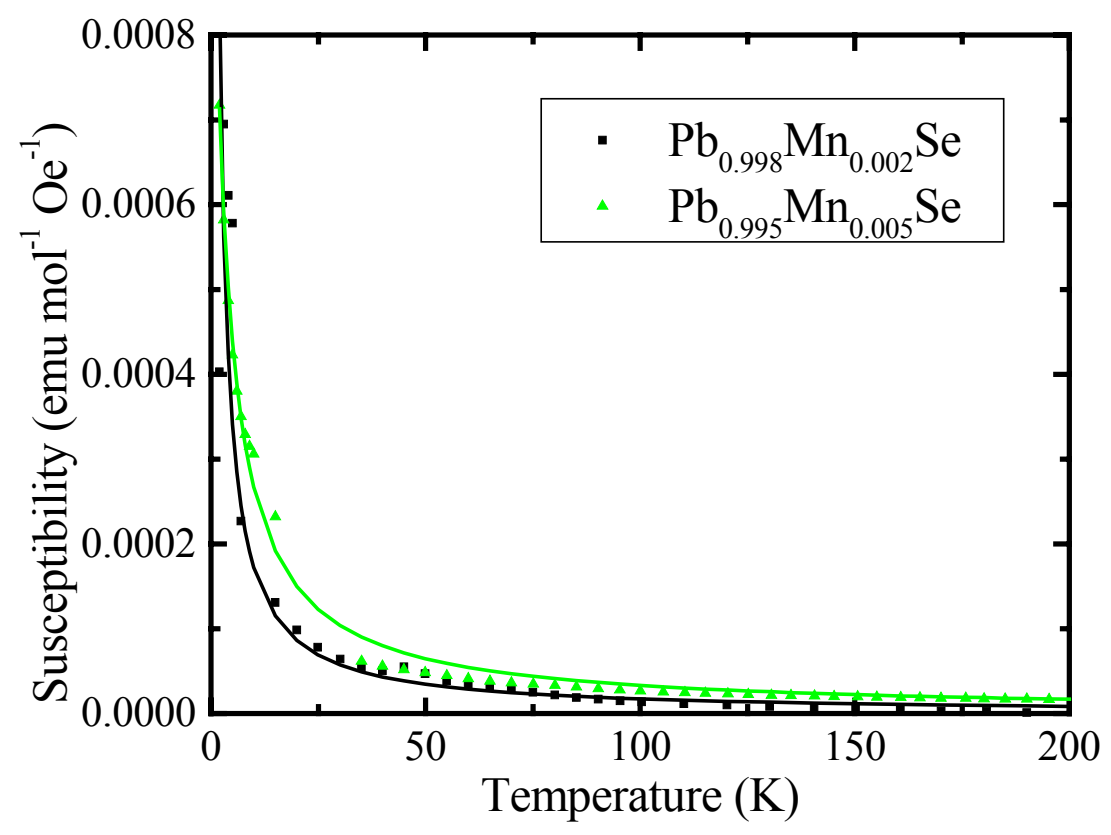

Figure S3. Susceptibility of $\mathrm{Pb}_{(1-\mathrm{x})} \mathrm{Mn}_{\mathrm{x}}$ Se corrugated nanoarrays $(\mathrm{x}=0.002$ and 0.005$)$ as a function of measuring temperature, indicating a presence of $\mathrm{Mn}^{2+}$ incorporated in nanoarrays.

The magnetic properties of the corrugated nanoarrays were studied by using the superconducting quantum interference device (SQUID) magnetometer. The susceptibility is fitted by a paramagnetic term $\chi_{\mathrm{p}}$, obeying the Curie-Weiss law, with an additional shift of a constant $\chi_{0}$. The temperature dependent susceptibility shown in Figure S3 is "corrected susceptibility" after a subtraction of the constant shift $\chi_{0}$ from the total (measured) susceptibility. These paramagnetic susceptibilities indicate that more $\mathrm{Mn}$ ions were introduced into the $\mathrm{Pb}_{0.995} \mathrm{Mn}_{0.005} \mathrm{Se}$ corrugated nanoarrays than that into the $\mathrm{Pb}_{0.998} \mathrm{Mn}_{0.002} \mathrm{Se}$ corrugated nanoarrays. The curves in Figure S3 are the best fitting and the fitted Mnconcentrations are 0.0007 and 0.0004 for $\mathrm{Pb}_{0.995} \mathrm{Mn}_{0.005} \mathrm{Se}$ and $\mathrm{Pb}_{0.998} \mathrm{Mn}_{0.002} \mathrm{Se}$ corrugated nanoarrays, respectively. The fitted Mn-concentration from the SQUID data is much smaller than that obtained from the ICP results. The Curie or Weiss temperature can also be obtained from the fitting of paramagnetic susceptibilities. The best fitting of the Weiss temperatures are $1.5 \mathrm{~K}$ and $0.1 \mathrm{~K}$ for $\mathrm{Pb}_{0.995} \mathrm{Mn}_{0.005} \mathrm{Se}$ and $\mathrm{Pb}_{0.998} \mathrm{Mn}_{0.002} \mathrm{Se}$ corrugated nanoarrays, respectively. The increase of Weiss temperature exhibits stronger antiferromagnetic interactions between the $\mathrm{Mn}$ ions in the $\mathrm{Pb}_{0.995} \mathrm{Mn}_{0.005}$ Se congregated nanoarrays. This result 
is consistent with bulk samples. ${ }^{2}$ The SQUID investigation implies that the Mn ions were incorporated into the $\mathrm{Pb}_{0.995} \mathrm{Mn}_{0.005} \mathrm{Se}$ corrugated nanoarrays.

\section{Reference:}

1. Z. L. Wang, "Transmission Electron Microscopy of Shape-controlled Nanocrystals and Their Assemblies", J. Phys. Chem. B 2000, 104, 1153.

2. M. Górska and J. R. Anderson, "Magnetic Susceptibility and Exchange in IV-VI Compound Diluted Magnetic Semiconductors", Phys. Rev. B 1988, 38, 9120. 